\title{
Suspected alimentary poisoning by aluminium phosphide in horses - a case report
}

\author{
Zuzana Široká1, Radek Melka², Alena Honzlová3, Radka Dobšíková4, Zdeňka Svobodová1 \\ ${ }^{1}$ University of Veterinary and Pharmaceutical Sciences Brno, Faculty of Veterinary Hygiene and Ecology, \\ Department of Animal Protection and Welfare and Public Veterinary Medicine, Brno, Czech Republic \\ ${ }^{2}$ Veterinary Center s.r.o., Susice, Czech Republic \\ ${ }^{3}$ State Veterinary Institute Jihlava, Department of Chemistry, Jihlava, Czech Republic \\ ${ }^{4}$ University of Veterinary and Pharmaceutical Sciences Brno, Faculty of Veterinary Hygiene and Ecology, \\ Department of Animal Breeding, Animal Nutrition and Biochemistry, Brno, Czech Republic
}

Received May 12, 2020

Accepted September 14, 2020

\begin{abstract}
In November 2018, a horse owner from the Czech Republic experienced the loss of two of his stallions in consequence of probable poisoning. The affected animals were kept in the stable only. The mares, which were grazing on pasture during the day, were not affected. All the animals were fed the same type of feed. The day before the animals became sick, between 15:00 to 19:00 $\mathrm{h}$ the stable was not secured against the entry of strangers. When the mares were brought back to the stable and all animals were closed and locked for the night, no signs of health issues were observed. In the following morning, the two stallions were found in lateral recumbency with seizure attacks. Despite symptomatic treatment (infusion with saline, flunixine meglumine, activated charcoal) and applications of myorelaxants, the symptoms persisted until the afternoon, and the owner agreed with euthanasia. Based on the clinical signs and pathological examination, poisoning by pesticides was suspected. The results of toxicological analyses revealed that carbofuran, metaldehyde, and strychnine were not responsible for the poisoning as they were not detected in the samples. The only positive finding was a high aluminium concentration in the stomach content, liver and kidney samples. Based on the observed symptoms and the high aluminium concentration, it was presumed that aluminium phosphide could be the source of poisoning. As there was no evidence of the possession of such product by the horse breeder and the stable stayed unsupervised for a few hours, intentional poisoning cannot be excluded.
\end{abstract}

Phosphine, pesticide, AlP

In November 2018, a horse owner from the Czech Republic experienced the loss of two of his stallions in consequence of probable poisoning (weights 350 and $500 \mathrm{~kg}$ ). Based on the very quick onset in more than one animal and the dominating clinical signs which were of neurological nature, the veterinarian suspected pesticide poisoning. The owner suspected intentional poisoning by an unknown person and the case was reported to the Police of the Czech Republic.

Based on the case circumstances, there was a suspicion of aluminium phosphide poisoning. Metal phosphides are also commonly used pesticides. They are mainly used as rodenticides, with aluminium phosphide also used as insecticide during grain storage. In the Czech Republic, zinc phosphide can be used only by registered farmers, aluminium phosphide can be applied only by certified disinfection, disinsection and deratisation (DDD) companies. Less information is available for aluminium phosphide than for zinc phosphide; but in general, it is assumed to have similar properties and effects as zinc phosphide. After ingestion, aluminium phosphide is hydrolysed by water/humidity and hydrochloric acid present in the stomach to a gaseous product phosphine $-\mathrm{PH}_{3}$, which is the active and poisonous agent (Peterson and Tallcott 2006; Gurjar et al. 2011; Hashemi-Domeneh et al. 2016). 
Phosphine is heavier than air and flammable (Peters on and Tallcott 2006; HashemiDomeneh et al. 2016). Its products (substituted phosphines and diphosphines) smell of decaying fish or garlic (some authors describe it also as rotten eggs or acetylene smell) (Gurjar et al. 2011). Unfortunately, the threshold for its detection is only around $2 \mathrm{ppm}$ in humans (Hashemi-Domeneh et al. 2016) but poisoning may occur even at lower concentrations. Phosphine is readily absorbed via mucosal membranes of the digestive tract and via lungs, then it is distributed by blood to all organs and later excreted mainly unchanged via lungs. For zinc phosphide it has been reported that part of the dose may be absorbed even without conversion to phosphine, being active in itself and causing delayed hepatotoxic and nephrotoxic effects (Peterson and Tallcott 2006; HashemiDomeneh et al. 2016). Zinc and aluminium that remain after phosphide decomposition are partially absorbed from the digestive tract as well (Gupta 2012).

The mechanism of action of phosphine includes reduction effects and interaction with sulphhydryl groups and subsequent inhibition of metalloenzymes. The most important is the inhibition of mitochondrial enzymes, mainly cytochrome oxidases, and thereby the inhibition of oxidative phosphorylation and energy production. During this process, reactive oxygen species are released contributing to cell damage and death. Phosphine is also known to partially inhibit acetylcholinesterase, but this is probably not clinically relevant (Peterson and Tallcott 2006; Hashemi-Domeneh et al. 2016). The lethal dose $\left(\mathrm{LD}_{50}\right)$ of zinc phosphide ranges between $20-40 \mathrm{mg} / \mathrm{kg}$. Similar values can be expected also for aluminium phosphide for which only $\mathrm{LD}_{50}$ for rats is known (oral $\mathrm{LD}_{50}$ $14 \mathrm{mg} / \mathrm{kg}$, inhalation $\mathrm{LC}_{50} 1 \mathrm{ppm}$ ) (Gangoli 1999). Clinical signs develop quickly. First signs may appear as early as within 15 min (vomiting), most of the symptoms are observed within $4 \mathrm{~h}$ after exposure. First signs involve anorexia, hypersalivation, depression, stomach distension, colic, vomiting, and retching with foul smelling breath. Digestive tract irritation and damage may be extensive and extremely painful, so the animals often react with vocalisation, anxiety or aggressiveness (Peterson and Tallcott 2006). After phosphine absorption, tachypnoe and dyspnoe are described, often also lung oedema and foaming from nostrils (both after inhalation of phosphine or as a result of exhalation of phosphine and direct effect on the alveoli). An acute respiratory distress syndrome may develop (Gurjar et al. 2001). Methaemoglobinaemia, Heinz bodies and cyanosis may occur (Hashemi-Domeneh et al. 2016). Phosphine affects cardiac and vascular tissues resulting in hypotension, congestive heart failure, myocarditis or pericarditis (Gurjar et al. 2011). Neurological signs start to be pronounced later with ataxia, weakness, recumbency, involuntary movements, teeth grinding, stool and urine release, tremors or even seizures and hyperthermia (Peters on and Tallcott 2006; Easterwood et al. 2010). Disseminated intravascular coagulation may occur as well. Delayed consequences after acute poisoning survival have been described for both zinc and aluminium phosphides, with liver or kidney damage or even failure 5 to 14 days after the acute phase (Peterson and Tallcott 2006; Gurjar et al. 2011).

Laboratory examination usually reveals methaemoglobinaemia, hypoxia, electrolyte changes, elevated liver enzymes, bilirubin and creatine kinase, metabolic acidosis and cholinesterase inhibition (Peterson and Tallcott 2006; Hashemi-Domeneh et al. 2016); also a decrease in glutathione may be detected (Gurjar et al. 2011). Dominant findings in pathological examination are hyperaemia and oedema of lungs, liver and kidney congestion, and pericarditis. Histological findings include renal tubular degeneration, focal myocardial necrosis and fatty degeneration of hepatocytes (Gfeller and Messonier 2004; Peterson and Tallcott 2006; Easterwood et al. 2010; Hashemi-Domeneh et al. 2016). Laboratory diagnosis can be made only from the gastric content which must be frozen and kept closed air-tight. Blood and urine analysis is not reliable as phosphine is rapidly oxidised to phosphite and hypophosphite (Gurjar et al. 2011). 
There is no antidote to this poison, only symptomatic and supportive measures are indicated. Gut decontamination with bicarbonate lavage, administration of activated charcoal, oxygen supply and ventilation support, fluid therapy to manage the mineral and acid-base imbalance, glucose, antioxidants (vitamin $\mathrm{C}$ and $\mathrm{N}$-acetylcysteine), intravenous lipid emulsion and anticonvulsant therapy are the main steps (Gurjar et al. 2011). A newly recommended measure is gastric lavage with coconut oil, which may protect gastric mucosa and prevent decomposition of aluminium phosphide tablet in stomach (Agrawal et al. 2015; Hashemi-Domeneh et al. 2016). Despite these interventions, mortality due to this poisoning is high, between $30-100 \%$ in humans depending on the dose ingested and the time elapsed between ingestion and the arrival to emergency unit (Gurjar et al. 2011; Agrawal et al. 2015).

To our knowledge, there is only one case of aluminium phosphide poisoning in horses described in scientific literature and it happened in the USA (Easterwood 2010). So reporting another case which also includes complementary information on aluminium levels in the tissues of affected animals can contribute to the current knowledge of pesticide poisonings.

\section{Case description}

Two stallions (horse No. 1, weight 350; and horse No. 2, weight $500 \mathrm{~kg}$ ) bred by a private owner were found ill one autumn morning. The affected animals were kept in the stable only. The mares of the same owner, which were grazing on pasture during the day, were not affected. All the animals were fed the same type of feed (hay, commercial feed mixture, commercial granulated feed, vegetables). The day before the animals became sick, between 15:00 to 19:00 $\mathrm{h}$ the stable was not secured against the entry of strangers with the owner being present in the nearby garden and family house. When the mares were brought back to the stable at around 19:00 h and all animals were closed and locked for the night, no signs of health issues were observed. The illness was discovered the next morning at around 8:00 h.

\section{Clinical signs}

Both affected horses showed apathy, decreased feed intake, hypothermia, hypotonia, and intense seizure attacks at 15 min intervals. They were lying in lateral recumbency and their skin and subcutaneous tissues were damaged. Bruises and haemorrhages were present in the skin, caused by mechanical damage during cramping, and the mucosas were anaemic. The horses were given activated charcoal, flunixine meglumine and saline infusion and were covered with blankets. Before lunchtime, the horses stopped eating completely, the seizure attacks became increasingly frequent with no reaction to the treatment with diazepam and barbiturates, and the owners agreed to have the affected stallions euthanized.

\section{Blood examination}

In the morning, immediately after finding the sick horses, a veterinarian living in the same village was called and blood samples from both affected horses were taken. Albumin, total protein, alkaline phosphatase (ALP), alanine aminotransferase (ALT), amylase, total bilirubin, blood urea nitrogen (BUN), creatinine, calcium, phosphorus, sodium, potassium and glucose levels were determined in blood plasma (VETSCAN ${ }^{\circledR}$ VS2 Chemistry Analyser, Abaxis Inc., USA). The results (Table 1) were available at lunch time and revealed an elevation in ALT and bilirubin, a significant elevation in glucose, a significant decrease in creatinine. In one horse, also total protein was decreased and in the other horse, decreased sodium and phosphorus were found. Other indices were within the reference range. 
Table 1. Results of the biochemical analysis of blood plasma.

\begin{tabular}{|c|c|c|c|c|}
\hline Indicator & Horse 1 & Horse 2 & Unit & Range \\
\hline ALB & 29 & 33 & $\mathrm{~g} / 1$ & $22-37$ \\
\hline ALP & 1.6 & 1.6 & $\mu \mathrm{kat} / 1$ & $0-2.8$ \\
\hline ALT & 0.9 & 1.0 & $\mu \mathrm{kat} / 1$ & $0.1-0.4$ \\
\hline 0.4AMY & 0.1 & 0.1 & $\mu \mathrm{kat} / 1$ & $0.1-0.3$ \\
\hline TBIL & 64 & 63 & $\mu \mathrm{mol} / 1$ & $3-35$ \\
\hline BUN & 5.3 & 6.0 & $\mathrm{mmol} / \mathrm{l}$ & $3.3-5.9$ \\
\hline $\mathrm{Ca}$ & 2.86 & 2.37 & $\mathrm{mmol} / \mathrm{l}$ & $2.25-2.99$ \\
\hline PHOS & $\underline{0.78}$ & 1.13 & $\mathrm{mmol} / \mathrm{l}$ & $1-1.81$ \\
\hline CREA & $\underline{67}$ & $\underline{65}$ & $\mu \mathrm{mol} / 1$ & $106-168$ \\
\hline GLU & 7.3 & 9.9 & $\mathrm{mmol} / \mathrm{l}$ & $4.2-6.4$ \\
\hline $\mathrm{Na}$ & $\underline{131}$ & 132 & $\mathrm{mmol} / \mathrm{l}$ & $132-142$ \\
\hline K & 4.6 & 3.8 & $\mathrm{mmol} / \mathrm{l}$ & $3.5-5.5$ \\
\hline $\mathrm{TP}$ & $\underline{55}$ & 61 & $\mathrm{~g} / 1$ & $60-80$ \\
\hline
\end{tabular}

ALB - albumin, ALP - alkaline phosphatase, ALT - alanin aminotransferase, AMY - amylase, TBIL - total bilirubin, BUN - blood urea nitrogen, $\mathrm{Ca}$ - calcium, PHOS - phosphorus, CREA - creatinine, GLU - glucose, $\mathrm{Na}$ - sodium, $\mathrm{K}$ - potassium, TP - total protein.

Elevated values marked in bold, decreased values marked by underlining
Necropsy was performed on the horses by the state veterinary pathologist at a local sanitation company 3 days after their death. Except for superficial injuries and effusion in the pericardium, only non-specific changes in the liver, lungs and intestinal mucosa such as hyperaemia and congestion were found. No samples were sent for histological examination. Samples of the liver, kidney and gastric content were sent to the State Veterinary Institute Jihlava for toxicological screening. As can be seen from the results (Table 2), neurotoxic pesticides suspected of having caused the poisoning

Table 2. Results of toxicology analyses.

\begin{tabular}{|c|c|c|c|c|c|c|}
\hline Source & Sample & Zinc (mg/kg) & Aluminium (mg/kg) & Carbofuran & Strychnine & Metaldehyde \\
\hline \multirow[t]{3}{*}{ Horse 1} & Gastric content & 75.6 & 387.2 & negative & negative & negative \\
\hline & Liver & - & 6.25 & negative & - & - \\
\hline & Kidney & - & 8.29 & - & - & - \\
\hline \multirow{3}{*}{ Horse 2} & Gastric content & 20.99 & 268.9 & negative & negative & negative \\
\hline & Liver & - & 2.17 & negative & - & - \\
\hline & Kidney & - & 5.23 & - & - & - \\
\hline \multirow[t]{5}{*}{ Feed } & Hay & 16.1 & 60.3 & - & - & - \\
\hline & Dry powdered feed & 30.1 & 2.04 & - & - & - \\
\hline & Dry granulated feed & 208.3 & 202.3 & - & - & - \\
\hline & Vegetables & 47.8 & 577.0 & - & - & - \\
\hline & Molasses & 36.7 & 34.2 & - & - & - \\
\hline
\end{tabular}

Liver and kidney values are given in $\mathrm{mg} / \mathrm{kg}$ of wet weight; the gastric content and feed samples are given in $\mathrm{mg} / \mathrm{kg}$ of dry matter. (Note: hay forms $95 \%$ of the diet.)

(carbofuran, strychnine or metaldehyde) were not detected in the animals' tissues. Analysis of the gastric content for phosphine could not be performed as too much time had elapsed between the horses' death and the necropsy and the gas had already evaporated. An indirect method of zinc and aluminium assessment was performed to determine whether metal phosphides were responsible for the poisoning. Levels of $\mathrm{Zn}$ and $\mathrm{Al}$ were assessed by ICP-OES (inductively coupled plasma optical emission spectrometry, Agilent 5100, Santa Clara, USA). Sample mineralisation for this analysis used nitric acid and hydrogen peroxide (nitric acid 69\% TraceSELECT, Honeywell, Charlotte, USA; hydrogen peroxide 30\% Lach-Ner, Neratovice, Czech Republic), equipment ETHOS Plus START D Microwave 
Digestion System (Sorisole, Italy). Zinc concentrations were within the reference range. The aluminium content in the liver of horse No. 2 was within the reference range, whereas in horse No. 1 it was elevated. The aluminium contents in the kidneys of both horses were substantially elevated.

\section{Discussion}

In the reported case, early signs of the poisoning were not observed as the horses were only found the next morning, many hours after the possible contact with the poison. Based on the severe neurological symptoms present concurrently in more than one horse, the veterinarian deemed an infectious disease as a less probable cause of the health issues and suspected pesticide poisoning. Carbofuran (insecticide that acts as an inhibitor of acetylcholine esterase), metaldehyde (molluscicide that decreases levels of the inhibitory GABA neurotransmitter), strychnine (rodenticide that inhibits receptors for the inhibitory glycine neurotransmitter) and zinc/aluminium phosphides (rodenticides/insecticides that are the inhibitors of oxidative phosphorylation) were considered (Peters on and Tallcott 2006; Gupta 2012). However, the clinical signs in our cases were non-specific. While apathy, recumbency, tremors and seizures are described for most of the above listed possible poisonings, hypothermia does not fit any. Another diagnostic problem was the lack of observed early clinical signs as the horses were found only many hours after possible poison ingestion.

The results of biochemical analyses (Table 1) of the plasma taken from both affected horses showed liver damage (bilirubin). Bilirubin can be elevated also due to haemolysis, but the haematological examination of blood was unfortunately not performed in this case. The ALT elevation can be a consequence of increased muscle work (seizures). Clinical examination revealed pale anaemic mucous membranes, so haemolysis cannot be excluded. The increased glucose concentration is evidence of a stressful situation for the horses and is often found as a consequence of trauma which was also described in this case (massive superficial damage due to intense cramps) (eClinPath 2019). Hyperglycaemia was described also as a laboratory finding during aluminium phosphide poisonings in humans in whom it is considered a negative prognostic sign (Mehrpour et al. 2008). Decreased creatinine is an atypical finding in this case, as it is typical for muscle tissue loss and immobility, whereas the affected horses were in a very good physical condition just one day before death and in the moment of sampling they were recumbent but had an increased muscle activity due to seizures (eClinPath 2019). Similarly, the decreased phosphorus is atypical, but as the phosphine is excreted mainly unchanged via exhalation (Hashemi-Domeneh et al. 2016), it usually does not contribute to plasma phosphorus concentrations. The results of this analysis are not in the agreement with the only other case of horse poisoning by aluminium phosphide described by Easterwood et al. (2010). In that case, the horses were found to have clinical signs of poisoning approximately $14 \mathrm{~h}$ after ingestion of feed treated freshly with aluminium phosphide (the protection/withdrawal period was not respected). Biochemical analysis revealed increased ALP, aspartate aminotransferase (AST), lactate and ammonia, and decreased glucose. Increased ammonia was due to liver damage, the affected horses suffered from hepatopathic encephalopathy. In our case study, blood was collected only at the beginning of the case investigation, when signs of encephalopathy were not seen, so ammonia concentrations were not evaluated. The ALP and AST may rise due to many reasons, lactic acidosis is connected with breathing difficulties and increased muscle work. Hypoglycaemia found in this case could not be explained by the authors, although it may sometimes appear in humans (Hashemi-Domeneh et al. 2016). In most cases, involving both humans (Gurjar et al. 2011; Agrawal et al. 2015) and animals, and also in our case, hyperglycaemia was described. 
In the case described by Eastewood et al. (2010), the lethality in the horses poisoned by aluminium phosphide exceeded $90 \%$ within $48 \mathrm{~h}$ after exposure, despite extensive treatment. In our case, we had a similar experience, the horses did not respond to the therapy (infusion, flunixine meglumine, activated charcoal, diazepam) and had to be euthanized.

The results of toxicological analyses revealed that carbofuran, metaldehyde and strychnine were not responsible for the poisoning as they were not detected in the samples. Based on the indirect method of assessment of zinc and aluminium which remain in the gastric content and tissues (mainly liver and kidney) even after death (Gupta 2012), non-physiological exposure to aluminium was proved.

Physiological values for the aluminium content in horse tissues are not mentioned in the literature. However, we obtained data from 8 healthy horses that had died due to other causes than poisoning and that were necropsied including a multi-metal analysis of their organs at the State Veterinary Institute Jihlava in the years 2014-2015. The healthy horses that were part of this internal monitoring had a mean aluminium concentration of $2.94 \pm 1.12 \mathrm{mg} / \mathrm{kg}$ wet weight (range 1.59-5.2 mg/kg of wet weight) in the liver, and $0.90 \pm 0.68 \mathrm{mg} / \mathrm{kg}$ of wet weight (range $0.084-1.93 \mathrm{mg} / \mathrm{kg}$ of wet weight) in the kidneys. The liver aluminium content (Table 2) of horse No. 2 was thus within the reference range, whereas in horse No. 1 it was approximately $\times 2$ higher than the mean value and slightly increased compared to the upper value of the range. The kidney aluminium concentration of horse No. 2 was approximately $\times 5$ higher compared to the average in non-poisoned horses; in horse No. 1 it was approximately $\times 8$ higher compared to the average. Both kidney values were much higher than the upper value of the reference range. A very interesting finding is also the ration of the liver and kidney aluminium contents which was approximately 3:1 (liver vs. kidney) in healthy horses, whereas in the affected horses it was inverted (3:4 in horse No. 1, and 2:5 in horse No. 2). We do not have an explanation for this, because data published by Anger et al. (2000) for humans indicated an increase of aluminium in the blood and liver after acute phosphide poisoning, however, without an increase in the kidneys and urine, probably due to the patient's rapid death.

In this case report, all horses were fed by the same type of feed at the same time of day, but only two of them fell sick. The horse feed consisted mainly of hay (95\%), other feeds formed less than $5 \%$ of daily intake (commercial feed mixture and granules, molasses and vegetables). Aluminium concentrations in the hay (the dominant part of feed) were approximately $\times$ 4-6 lower than in the gastric content of the affected horses (Table 2). As can be seen from the zinc concentrations (Table 2), the gastric contents and those in the feed were comparable for horse No. 2 and just slightly increased in the gastric sample of horse No. 1 compared to the feed, which means that the feed was the main source of zinc determined in both animals. The zinc concentration was not elevated, and so zinc phosphide was not responsible for the horses' sickness.

Based on the comparison of aluminium concentrations in the horses' gastric content and the feed (especially in hay as the predominant part of the feed), the feed was not responsible for the increase in aluminium in the horses' tissues. High aluminium concentrations in the internal organs prove that aluminium was absorbed into the horses' bodies and that the source was outside the feed. The most probable situation was poisoning caused by an aluminium phosphide-based pesticide product. The cause of tissue damage, neurological symptoms and consequent death of the animals was not the aluminium itself but the phosphine released from phosphide in the stomach of the affected animals. Aluminium concentration assessment is only an indirect method of proving the exposition of animals to an aluminium phosphide bait. The source of poisoning was not uncovered but as only the horses kept in the unprotected stable during the day were affected, and as the owner did not possess any phosphide and none of it was found on the farm, intentional poisoning by an unknown person was suspected. 
Unfortunately, there was not enough evidence for the police investigation and the case went cold.

To our knowledge, there is very little information regarding the aluminium concentrations determined in the tissues after a poisoning by aluminium phosphide. The only case of a horse poisoning by phosphide described in the literature by Easterwood et al. (2010) does not mention this diagnostic possibility. Studies by Garry et al. (1993) or Anger et al. (2000) mentioned this possibility in humans, however, this procedure is not common. We believe our approach may help diagnose the source of the poisoning where analysis of phosphine is not possible, e.g. due to a time delay or improper storage of the stomach content sample.

\section{Acknowledgement}

The authors thank the Internal Creative Agency of the University of Veterinary and Pharmaceutical Sciences Brno (project FVHE/Vecerek/ITA2019) for the financial support.

\section{References}

Agrawal VK, Bansal A, Singh RK, Kumawat BL, Mahajan P 2015: Aluminium phosphide poisoning: Possible role of measures in the absence of specific antidote. Indian J Crit Care Med 19: 109-112

Anger F, Paysant F, Brousse F, Le Normand I, Develay P, Galliard Y, Baert A, Le Gueut MA, Pepin G, Anger JP 2000: Fatal aluminium phosphide poisoning. J Anal Toxicol 24: 90-92

Easterwood L, Chaffin MK, Marsh PS, Porter B, Barr C 2010: Phosphine intoxication following oral exposure of horses to aluminium phosphide-treated feed. JAVMA 236: 446-450

eClinPath - online textbook on Veterinary Clinical Pathology, Cornell University. Available at: http://eclinpath. com/. Accessed December 5, 2019.

Gangolli S (Ed.) 1999: The dictionary of substances and their effects, volume 1. The Royal society of chemistry, Cambridge, $889 \mathrm{p}$.

Garry VF, Good PF, Manivel JC, Perl DP 1993: Investigation of a fatality from non occupational aluminium phosphide exposure: measurement of aluminium in tissue and body fluids as a marker of exposure. J Lab Clin Med 122: 739-747

Gfeller RW, Messonier SP 2004: Small animal toxicology and poisonings. Mosby, St. Louis, 498 p.

Gupta RD (Ed.) 2012: Veterinary toxicology, second edition. Academic Press, London, 1438 p.

Gurjar M, Baronia AK, Azim A, Sharma K 2011: Managing aluminium phosphide poisonings. J Emerg Trauma Shock 4: 378-384

Hashemi-Domeneh B, Zamani N, Hassanian-Moghaddam H, Rahimi M, Shadnia S, Erfantalab P, Ostadi A 2016: A review of aluminium phosphide poisoning and a flowchart to treat it. Arh Hig Rada Toksikol 67: 183-193

Mehrpour O, Alfred S, Shadnia S, Keyler DE, Soltaninejad K, Chalaki N, Sedaghat M 2008: Hyperglycemia in acute aluminium phosphide poisoning as a potential prognostic factor. Hum Exp Toxicol 27: 591-595

Peterson ME, Talcott PA 2006: Small animal toxicology. Saunders Elsevier, St. Louis, 1190 p. 